\title{
Data format notations for technical communication among hardware and software engineers
}

\begin{abstract}
Communication among software and hardware engineers plays an important and significant role in successful completion of projects in the environments that hardware and software engineers cooperate to develop common products. In these environments communication is inevitable because both hardware and software engineers need to be aware of each otherôs main challenges and concerns with respect to the common project. The need for this communication is perceived to be even more in developing hardware-software interfaces and also in clarification of types and formats of the data that will be transferred between hardware and software components. In this paper, three different notations are proposed to help software and hardware engineers communicate each other in written and document-based way. By using these notations which are understandable by hardware and software engineers, the requirements relating to the data types and data formats will be depicted in uniform, detailed and accurate forms of documents. These notations that will be drawn by either hardware or software engineers in requirements phase, will describe the type and format of any interaction data at any size.
\end{abstract}

Keyword: Technical communication; Communication notation; Requirement; HW/SW systems; Hardware/software engineers 